\title{
Nachhaltigkeitsberichte mit Biss
}

\author{
Aus der Kritik an schwacher Nachhaltigkeits- \\ leistung von Unternehmen und der lückenhaften \\ Unternehmensverantwortung ist das Konzept \\ der Gemeinwohlökonomie entstanden. \\ Im Folgenden soll erörtert werden, durch welche \\ Wirkungsprinzipien sie wertvolle Impulse für \\ die künftige Berichterstattung und den Gesetz- \\ geber liefert. Von Gerd Hofielen
}

M it Nachhaltigkeitsberichten können Unternehmen Rechenschaft darüber ablegen, wie der Geschäftsbetrieb auf ihre Stakeholder - inklusive Gesellschaft und Natur - wirkt. So kann für Außenstehende einsichtig werden, wie ein Unternehmen seine Gewinne erzielt, und das Unternehmen kann zeigen, auf welche Weise die Verantwortung für eine nachhaltige Gestaltung der Geschäftspraxis wahrgenommen wird.

\section{Die kritikwürdige Situation der Nachhaltig- keitsberichterstattung}

Die Nachhaltigkeitsberichterstattung hat nach meiner Einschätzung allerdings die tatsächlichen Nachhaltigkeitsleistungen der Unternehmen bisher nicht entscheidend verändert. Wesentliche Kritikpunkte an der herkömmlichen Nachhaltigkeitsberichterstattung sind:

- Die Güte des Nachhaltigkeitsberichts eines Unternehmens ist nicht identisch mit der tatsächlichen Nachhaltigkeitsleistung.

- Die Unternehmen sind nicht verpflichtet, ein vollständiges Abbild der Ressourcenbeanspruchung zu geben und lassen die Berichte nur zum Teil auditieren.

- Die Berichte sind unübersichtlich, nehmen keine Bewertung vor und sind nur nach aufwendigen Recherchen zwischen Unternehmen vergleichbar.

- Auch die vielfältigen Nutzen, die das Unternehmen schafft, werden nicht verständlich bewertbar dargestellt.

- An die berichtete Nachhaltigkeitsleistung sind keine rechtlichen Folgen geknüpft, weder Anreize für besonders gute noch Sanktionen für unzulängliche Praktiken.

Damit ist eine Rechenschaftslegung gegenüber der Öffentlichkeit nur mangelhaft möglich.

Dieser Zustand der Nachhaltigkeitsberichterstattung ist nicht zufällig, sondern drückt den gesellschaftlichen Konsens in der Bundesrepublik Deutschland aus. Die Interessenorganisationen der Wirtschaft und die staatlichen Organe scheuen bis heute vor einer übersichtlichen, aussagefähigen und leicht vergleichbaren Berichterstattung über die Unternehmensverantwortung zurück. Die CSR-Richtlinie, eine Initiative der EU-Kommission (vgl. Beitrag von Ebinger in diesem Heft), wagte sich auf dieses Terrain vor. Der deutsche Gesetzgeber hat ihre Umsetzung auf das von der EU vorgegebene Mindestniveau reduziert.

Es ist eine Prämisse der CSR-Richtlinie, dass Transparenz und Vergleichbarkeit der Nachhaltigkeitsberichte in einer gesteigerten Nachhaltigkeitsleistung in der Praxis resultieren. Die Pflicht zur Veröffentlichung erfordert die Erfassung der Tatbestände und soll dazu führen, dass die Leitungspersonen und -organe des Unternehmens sich damit befassen. Bis zu einem gewissen Grad kann dies Wirkungen im Unternehmen entfalten. Aber die prekäre Situation der Menschenrechte in den internationalen Lieferketten und der Zustand der ökologischen Systeme legen nahe, dass zum bloßen Berichten viel mehr hinzukommen muss, um den erforderlichen Verantwortungswillen in die Unternehmen zu bringen.

Bislang werden Nachhaltigkeitsberichte vorwiegend mit einer defensiven Haltung erstellt, um die Geschäftsmodelle abzusichern und Reputationsschäden zu vermeiden. Diese Situation wird von vielen zivilgesellschaftlichen Organisationen und einer zunehmenden Anzahl von fortschrittlichen Unternehmen beklagt. Die konventionelle Wirtschaft wird als die Verursacherin der Probleme wahrgenommen und nicht als Teil der Lösung.

\section{Der Vorschlag der Gemeinwohlökonomie}

Vor diesem Hintergrund hat die Gemeinwohlökonomie (GWÖ) ein Modell vorgelegt, das geeignet ist, die Herausforderungen eines nachhaltigen Wirtschaftens dort sichtbar zu machen, wo sie angepackt werden können: in den Entscheidungen der Unternehmensleistungen [1]. Das ethische Niveau der Unternehmensleistung sichtbar zu machen ist ein, wenn nicht der, entscheidende Beitrag der GWÖ für den Übergang von der konventionellen zur zukunftsfähigen Wirtschaft. Das vorgeschlagene Instrument einer zutreffenderen Rechenschaftslegung ist die Gemeinwohlbilanz. Bisher haben rund 500 Unternehmen eine Gemeinwohlbilanz erstellt und veröffentlicht [2].

Die Gemeinwohlbilanz erfasst die Nachhaltigkeitsperformance in ganzheitlicher Sicht und bewertet diese nach dem Beitrag zu grundlegenden humanistischen Werten. Sie wird 
vollständig auditiert und das Audit-Testat muss mit dem Bericht auf den Internetseiten des Unternehmens und der GWÖ e. V. veröffentlicht werden. Das Audit-Testat wird auf einer DIN-A4 Seite ausgewiesen und ermöglicht die schnelle und leichte Vergleichbarkeit und den gezielten Einstieg in den detaillierten Bericht. Wie glaubt die GWÖ erreichen zu können, dass mit dem Berichten auch die Verantwortungsübernahme und praxiswirksame Veränderungen verstärkt werden?

\section{Die Wirkungsprinzipien der Gemeinwohl- bilanzen}

Das Modell der Gemeinwohlökonomie setzt auf diverse Wirkungsprinzipien, um aussagefähige Berichte und mehr Nachhaltigkeit in der Praxis zu erreichen.

- Verfassungswerte: Der Gemeinwohlbericht bezieht die Unternehmensaktivitäten auf Verfassungswerte, die auch im Grundgesetz Deutschlands verankert sind: Menschenwürde, Solidarität und soziale Gerechtigkeit, ökologische Nachhaltigkeit, Transparenz und demokratische Mitwirkung. Im Bericht wird dargestellt, wie die wirtschaftliche Leistung zu den Grundwerten beiträgt. Damit soll erreicht werden, dass die unternehmerische Prosperität im Einklang steht mit gesellschaftlicher Prosperität.

I Ganzheitlichkeit: Die Wirkungen der Unternehmenspraktiken werden ganzheitlich betrachtet, in Bezug auf alle Stakeholder und alle Werte. Stakeholder, in der GWÖ Berührungsgruppen genannt, sind Lieferanten, Finanzpartner, Eigentümer, Mitarbeitende, Kund/innen und die gesellschaftlichen Systeme. Damit wird das Unternehmen als Akteur mit gesellschaftlicher Wirkung erkennbar. Es wird zum Beispiel deutlich, wie die Menschenrechte in der Lieferkette beachtet werden, wie die Produkte in der Gesellschaft wirken und wie die Gehaltsspreizung im Unternehmen aussieht.

I Bewertung: Der Umgang mit den Berührungsgruppen wird in der Gemeinwohlbilanz auch bewertet und leicht vergleichbar dargestellt. Es wurde ein Punktesystem entwickelt, das die Performance pro Wert und Berührungsgruppe auf einer Skala von „erste Schritte“ bis „vorbildlich“ beschreibt und die Selbstbewertung des Unternehmens ermöglicht. Je stärker die Werte in einer Berührungsgruppe umgesetzt werden, desto höher ist die Punktebewertung. Das prozentuale Ergebnis wird für 20 Themen auf einer DIN-A4 Seite übersichtlich berichtet. Damit wird Greenwashing vermieden und es entsteht eine leicht zugängliche Vergleichbarkeit, die vor allem für Geldgeber/innen und Konsument/innen hilfreich ist.

I Ethische Orientierung: Das Bewertungssystem der GWÖ verknüpft die Praxis der Nachhaltigkeitsleistung mit normativen Zielen eines ethischen Wirtschaftsmodells. Ethik im Sinne der GWÖ bedeutet, bei unternehmerischen Praktiken den Schaden für Menschen und Natur gering zu halten oder abzuwenden und nützliche Beiträge für die Berührungsgruppen zu leisten. Die ethischen Vorstellungen im
GWÖ-Modell sind öffentlich zugänglich und werden von den Anwender/innen weiterentwickelt. Unternehmen mit höherer ethischer Sorgfalt, die zu stärkeren Leistungen in der Praxis führt, erreichen eine höhere Punktzahl. So wird zum Beispiel das Prinzip der innerbetrieblichen Transparenz und Mitwirkung positiv gesehen und je mehr davon feststellbar ist, desto mehr Wertungspunkte können erreicht werden. Wie ein Unternehmen dies macht, bleibt jedoch der Gestaltungsfreiheit der Beteiligten überlassen.

I Auditierung: Die Gemeinwohlbilanzen werden verpflichtend vollständig auditiert und das erreichte Punkteergebnis wird pro Berührungsgruppe und Wert in einem Testat festgehalten und in den Gemeinwohlbericht eingefügt. Die vollständige Überprüfung der Berichte und der Punktebewertung durch externe Auditor/innen schafft Vertrauen in die veröffentlichten Gemeinwohlbilanzen und das Prinzip der Transparenz ermöglicht allen, die an einem Unternehmen interessiert sind, den Einblick.

Mit diesen fünf Prinzipien - Verfassungswerte, Ganzheitlichkeit, Bewertung, ethische Orientierung und Auditierung wird ein Niveau von Transparenz erreicht, das aussagefähig ist. Marktteilnehmer/innen wie etwa Verbraucher/innen, öffentliche Beschaffer und Mitunternehmen erhalten eine nützliche Orientierung und können neben den konventionellen Kriterien wie Produktqualität, Lieferfähigkeit und Preisen auch den Gemeinwohlbeitrag von Unternehmen bei Kaufentscheidungen berücksichtigen. Damit kommen die Märkte näher an das Ideal der Transparenz, das für die Funktionsfähigkeit einer Marktwirtschaft grundlegend ist.

Die Gemeinwohlbilanz macht sichtbar, wie die Erfolge der Wirtschaft zum gesellschaftlichen Wohlstand beitragen. Es wird transparent, wie das Unternehmen den Gewinn erzielt und wie die wirtschaftlichen Entscheidungen die Lebensqualität aller Stakeholder in einer werteorientierten, gesellschaftlichen Perspektive beeinflussen. Die transparente Bewertung der Gemeinwohlökonomie bietet ein Steuerungsinstrument, das nützlich ist, um die schädigenden Konsequenzen wirtschaftlicher Tätigkeiten zu reduzieren und die positiven Wirkungen zu verstärken.

Drei aktuelle Studien (vgl. Giesenbauer et al. 2018, Heidbrink et al. 2018, Sanchis et al. 2018) haben dem Instrument der Gemeinwohlbilanz bescheinigt, dass es ein höheres Ambitionsniveau hat als bisherige CSR-Instrumente, dass es das Potenzial hat, zu einer Postwachstumsökonomie beizutragen, und eine valide soziale Innovation darstellt, die es wert ist, institutionalisiert zu werden.

\section{Rechtsfolgen und Skalierung nachhaltigen Wirtschaftens}

Wenn die Gemeinwohlbilanzierung wirtschaftliche und gesellschaftliche Bedeutung erlangen soll, sind Rechtsfolgen an den Gemeinwohlbeitrag zu knüpfen. So, wie die Richtlinien für die Steuerzahlung eines Wirtschaftsteilnehmers gesetzlich 
festgelegt werden, müsste auch eine gesetzliche Orientierung geschaffen werden, wie eine höhere Gemeinwohlleistung belohnt und eine niedrigere sanktioniert wird. Hierfür müssen die nützlichsten und wirkungsvollsten Festlegungen in einer breiten gesellschaftlichen Meinungsbildung noch ermittelt, in der Praxis überprüft und entsprechend angepasst werden.

Der Vordenker der Gemeinwohlökonomie Christian Felber (2018) ist der Auffassung, dass perspektivisch ein einheitliches Verfahren zur Nachhaltigkeitsberichterstattung entwickelt werden müsse. Neben der Finanzbilanz, die unter anderem zur Steuerfestsetzung dient, sollte eine einheitliche Berichterstattung entstehen, die zur Grundlage von Rechtsfolgen werden kann.

Gesetzliche Maßnahmen könnten bereits erprobte Instrumente verwenden wie etwa Forschungsförderung, Förderung der Gemeinwohlbilanzierung und der Gemeinwohl-Audits von Unternehmen und Gemeinden, Anerkennung von Gemeinwohlbilanzen beim Zugang zur öffentlichen Beschaffung, spezielle Kreditprogramme, reduzierte Steuersätze oder Zölle.

Die Begründung für eine präferierte Behandlung von staatlicher Seite sind die erhöhten Kosten und die ethische Sorgfalt der gemeinwohlbilanzierenden Unternehmen. Sie trachten danach, die üblicherweise externalisierten Kosten der Nutzung von Naturleistungen zu internalisieren, das heißt sie reduzieren, kompensieren oder substituieren die Leistungen der Natur in ihren Wertschöpfungsprozessen. Sie machen zudem die Beachtung der Menschenwürde bei Lieferant/innen zum Kriterium ihrer Kaufentscheidungen. So entsteht neben dem direkten Nutzen durch die Produkte und Dienstleistungen ein indirekter, sekundärer Nutzen für die Natur und die gesellschaftlichen Systeme.

Die Belohnungen für Gemeinwohlbilanzierungen würden wichtige Signale an konventionell wirtschaftende Unternehmen senden, die Lieferant/innen und Mitarbeiter/innen vorwiegend als Kostenfaktor begreifen und die Leistungen von Natur und Gesellschaft überwiegend externalisieren. Da diese Unternehmen finanziell getrieben sind, würden sie finanzielle Anreize sehr gut verstehen.

\section{Die Verbreitung der Gemeinwohlökonomie}

Der Beitrag der Gemeinwohlökonomie kommt aus der Gesellschaft, von Bürger/innen und Unternehmer/innen, die an einer Wirtschaft interessiert sind, die unternehmerischen Erfolg mit gesellschaftlicher Prosperität verbindet. Sie erhebt keinen Anspruch auf Vollkommenheit, wird permanent weiterentwickelt und möchte in der demokratischen Auseinandersetzung um die zukunftsorientierte Wirtschaft Impulse geben. Dieser Impuls wird von vielen Pionierunternehmen und Kräften in der Gesellschaft bereits aufgegriffen. Nicht zuletzt vom Europäischen Wirtschafts- und Sozialausschuss (EWSA), der mit einer Mehrheit von $86 \%$ beschlossen hat: „Nach Auffassung des EWSA sollte das Gemeinwohlökonomie-Modell so- wohl in den europäischen als auch die einzelstaatlichen Rechtsrahmen integriert werden. Ziel ist es, die Verwirklichung des Binnenmarkts über eine verstärkt ethische Wirtschaft voranzubringen, die auf europäischen Werten und der Wahrnehmung gesellschaftlicher Verantwortung gründet und diese synergetisch untermauert" [3].

\section{Anmerkungen}

[1] Die weltweit agierende Gemeinwohl-Ökonomie-Bewegung wurde 2010 ins Leben gerufen. Sie basiert auf den Ideen von Christian Felber. Aktuell umfasst sie weltweit rund 11.000 Unterstützer/innen, mehr als etwa 43.000 Aktive in über 150 Regionalgruppen, $31 \mathrm{GWÖ-Vereine,} 500$ bilanzierte Unternehmen und andere Organisationen, knapp 60 Gemeinden und Städte sowie 200 Hochschulen weltweit, die die Vision der Gemeinwohl-Ökonomie verbreiten, umsetzen und weiterentwickeln. Weitere Informationen unter www.ecogood.org.

[2] Siehe dazu Liste der GWO-Berichte unter www.balance.ecogood. org/gwoe-berichte.

[3] Siehe dazu EWSA-Stellungnahme zum Thema (ECO/378 - EESC-201502060-00-00-AC-TRA (ES) 1/611) unter www.eesc.europa.eu/en/ our-work/opinions-information-reports/opinions/economy-commongood\#downloads.

\section{Literatur}

Felber, C. (2018): Von der Vielfalt wirkungsloser CSR-Instrumente zu einer rechtsverbindlichen Ethik-Bilanz. www.hm-practices.org/blog/von-dervielfalt-wirkungsloser-csr-instrumente-zu-einer-rechtsverbindlichen-ethikbilanz-fu\%CC\%88r-unternehmen/

Giesenbauer, B./Müller-Christ, G.: Die Sustainable Development Goals für und durch KMU. Ein Leitfaden für kleine und mittlere Unternehmen. Bremen: Universität Bremen.

Heidbrink, L. et al. (2018): Schlussbericht für das Verbundprojekt Gemeinwohl-Ökonomie im Vergleich unternehmerischer Nachhaltigkeitsstrategien (GIVUN). Flensburg: Europa-Universität Flensburg.

Sanchis, J.R./Campos, V./Ejarque, A. (2018): Analyzing the Economy for the Common Good Model. Statistical Validation of its Metrics and Impacts in the Business Sphere. Valencia: Universität Valencia.

\section{AUTOR + KONTAKT}

Gerd Hofielen wurde 2008 von der Siemens AG beauftragt, an der internen Kampagne zur Verbreitung korruptionsfreier Geschäftspraktiken mitzuwirken. $\mathrm{Er}$ ist Gemeinwohlberater und arbeitet im Matrix-Entwicklungsteam der GWÖ mit.

Humanistic Management Practices $\mathrm{GmbH}$, gemeinnützig, Matterhornstr. 47, 14129 Berlin. Tel.: +49 178 2368814, E-Mail: gerd.hofielen@hm-practices.org, Website: https://www.hm-practices.org/

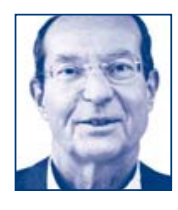

